\title{
Stereological Analysis of Mitochondria and Smooth Endoplasmic Reticulum Distribution in Human Oocytes at Prophase I
}

\author{
Luís A*, Rocha E*, Oliveira E*, Silva J**, Barros A*****, Sousa $\mathrm{M}^{*}$ \\ * Lab Cell Biology, ICBAS, University of Porto, Largo Prof. Abel Salazar 2, 4099-001 Porto, \\ Portugal \\ ** Centre for Reproductive Genetics A. Barros, Avenida do Bessa 591, $1^{\circ}$ dto frente, 4100-009 \\ Porto, Portugal \\ *** Dept. Genetics, Fac. Medicine, University of Porto, Alameda Prof. Hernâni Monteiro 4200 - \\ 319 Porto, Portugal
}

The structure of human oocytes at the different maturation stages is known based on descriptive studies of its ultrastructural composition [1-2]. The tridimensional quantitative ultrastructural analysis of human oocytes would be important for understanding folliculogenesis and establishing adequate methods of in vitro oocyte maturation. In this study, stereological methods were applied to quantify the distribution of mitochondria (M) and smooth endoplasmic reticulum (SER) in oocytes at the germinal vesicle (VG) stage of prophase I.

Under patient informed consent, immature GV oocytes collected during ICSI treatments were processed for electron microscopy. Five GV oocytes were completely serially sliced with a diamond knife in a ultramicrotome. Every $15 \mu \mathrm{m}$ sections were observed in a JEOL-100CXII transmission electron microscope at $60 \mathrm{kV}$ [3]. Photographs were taken following a systematic sampling grid and printed at x15900. The relative volume $(\mathrm{Vv})$ of each organelle was obtained by using classical manual stereological techniques based on point-counting, and applying the formulae $\mathrm{Vv}$ (organelle, oocyte) $=[$ number of points (organelle)/ number of points (oocyte)]x100 (\%) [4]. Each oocyte was divided into three regions from the oolema up to the cell center: cortex $(5 \mu \mathrm{m})$, subcortex $(5-10 \mu \mathrm{m})$ and inner cytoplasm $(10-60 \mu \mathrm{m})$, and the same procedure was adopted. Statistical analysis was performed with Microsoft Excel 2003 and SPSS 14.0. Results are presented as mean, standard error of the mean (SEM) and coefficient of variation (CV).

The Vv occupied by the organelles - M, SER isolated tubules (SER-IT), SER small vesicles (SER-SV), SER medium vesicles (SER-MV), SER large vesicles (SER-LV) and SER very large vesicles (SER-VLV) - in oocyte cortex, subcortex and inner cytoplasm was calculated (Table 1). Means of Vv (organelle,cortex/subcortex/inner cytoplasm) were compared with ANOVA $(\alpha=0.05)$. Post-hoc comparisons with Sheffé procedure was used as a subsequent analysis for significant ANOVAs. Concerning the three different ooplasm regions, ANOVA revealed significant differences for mitochondria, SER-VLV and SER-LV. These organelles occupied a significant higher relative volume in the oocyte inner cytoplasm - mitochondria: 7.66\%, SERVLV: $12.51 \%$, SER-LV: $0.26 \%$ - than in the cortex - mitochondria: $3.5 \%$, SER-VLV: $1.11 \%$, SER-LV:0,1\%.

Stereological analysis revealed that in human immature GV oocytes, mitochondria, SER-VLV and SER-LV displayed a preferential spatial distribution in the central region of the ooplasm. Additionally, we first described SER-VLV (Figure 1). Both findings may underlie the 
explanation for common oocyte abnormalities, particularly organelle migration towards the cortex and development of giant vacuoles, respectively.

\section{References}

[1] El-Shafie et al. An Atlas of the Ultrastructure of Human Oocytes. Parthenon Publishing Group, New York. 2000.

[2] Sousa M et al. Mol Hum Reprod 1997; 3, 83-90.

[3] Tesarik et al. Hum Reprod 1994; 9, 511-518.

[4] Freere and Weibel. J Royal Microsc Soc 1967;87: 25-34.

Table 1. Relative volume ( $\mathrm{Vv})$ of organelles in the oocyte, cortex, subcortex and inner cytoplasm.

\begin{tabular}{ccccc}
\hline Organelle & Vv (organelle, oocyte) & Vv (organelle, cortex) & $\begin{array}{c}\text { Vv (organelle, } \\
\text { subcortex) }\end{array}$ & $\begin{array}{c}\text { Vv (organelle, inner } \\
\text { cytoplasm) }\end{array}$ \\
\hline M & $6.29 \pm 0.64(0.20)$ & $3.50 \pm 0.62(0.35)$ & $6.50 \pm 0.63(0.19)$ & $7.66 \pm 1.28(0.33)$ \\
SER-VLV & $6.97 \pm 0.95(0.27)$ & $1.11 \pm 0.34(0.62)$ & $1.72 \pm 0.69(0.81)$ & $12.51 \pm 2.19(0.35)$ \\
SER-LV & $0.20 \pm 0.02(0.2)$ & $0.10 \pm 0.02(0.39)$ & $0.18 \pm 0.04(0.46)$ & $0.26 \pm 0.03(0.24)$ \\
SER-MV & $0.20 \pm 0.10(0.98)$ & $0.12 \pm 0.02(0.31)$ & $0.13 \pm 0.07(1.13)$ & $0.26 \pm 0.13(1.00)$ \\
SER-SV & $1.46 \pm 0.11(0.15)$ & $1.46 \pm 0.29(0.40)$ & $1.29 \pm 0.24(0.37)$ & $1.44 \pm 0.20(0.29)$ \\
SER-IT & $4.12 \pm 1.20(0.58)$ & $4.38 \pm 1.53(0.70)$ & $3.97 \pm 1.00(0.50)$ & $3.89 \pm 1.08(0.56)$ \\
\hline
\end{tabular}

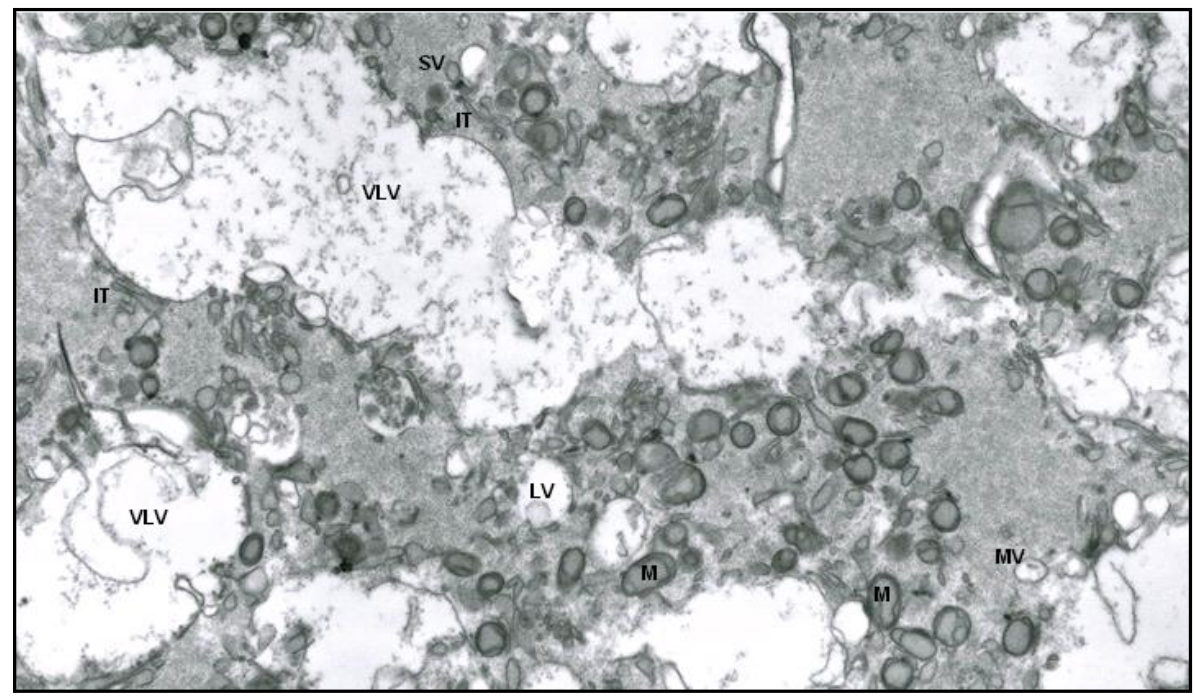

Figure 1. Photograph at the oocyte inner cytoplasm showing SER very large vesicles (VLV), SER small vesicles (SV), SER medium vesicles (MV), SER large vesicles (LV), SER isolated tubules (IT) and mitochondria (M) (15900x). 